\title{
General Analysis of Cube Satellite Technology: An Overview
}

\author{
Ritwik Sinha \\ Department of E.C.E. \\ Ajay Kumar Garg Engineering \\ College (Affiliated to AKTU U.P., \\ Lucknow) \\ Ghaziabad, 201009, India
}

\author{
Ananya Verma \\ Department of E.N. \\ Ajay Kumar Garg Engineering \\ College (Affiliated to AKTU U.P., \\ Lucknow) \\ Ghaziabad, 201009, India
}

\author{
Sachin Choudhary \\ Department of E.C.E. \\ Ajay Kumar Garg Engineering \\ College (Affiliated to AKTU U.P. \\ Lucknow) \\ Ghaziabad, 201009, India
}

\begin{abstract}
CubeSat technology has become a tool to encourage engineering collaboration, to train students providing them with a platform for real-world space exploration. This provides advancements in the aerospace industry as well. These satellites are made for a rather specific purpose than a conventional heavyweight satellite thereby reducing the cost. This paper discusses Cube Satellites, their design, salient features along with different applications and advancements made in the field of CubeSat technology thus providing an overview of a general analysis of CubeSat technology.
\end{abstract}

\section{Keywords}

Cube Satellite Technology, Space System

\section{INTRODUCTION}

Developing CubeSat standard, typically a picosatellite standard is encouraging engineering collaboration by becoming a tool to train students with real-world satellite experience. This also encourages advancement in the aerospace industry. Development of satellites at the CubeSat level throws light upon increasing opportunities for access to space with quicker development times. P-POD or The PolyPicosatellite Orbital Deployer along with CubeSat standard has an important role to play to provide access to space.

Many universities, companies, and government organizations are interested in CubeSat in order to perform valuable scientific experiments and missions seeing the upcoming launches. CubeSat technology offers a great platform for budding engineers and scientists.

Observing the recent advancements and developments in space technology it can be said that these are aimed at doing more for less cost which in turn leads to "Smaller, Cheaper, Faster, Better" space missions.

A better insight into the rationale and procedures for testing a larger satellite is provided by "test as you fly, fly as you test" approach. This has been quite effective since the testing required at CubeSat level is similar to actual launch provider, therefore, university facilities are usually adequate to support testing of an entire CubeSat system instead of only component level testing in case of larger spacecraft system.

Now in part-II, the paper discusses the designing of the satellite followed by sources of heat for general space system in part-III.

Part-IV discusses the applications. Furthermore, the paper describes the developed CubeSat in the subsequent part concluding with the salient features and limitations in part-VI and part-VII respectively.

\section{DESIGN}

\subsection{An Overview}

It takes about years to prepare a standard spacecraft but CubeSats being relatively inexpensive, small and weighing just a few kilograms can be made ready to launch in a matter of months. Standard CubeSats are made to provide 1 litre of useful volume $(10 \times 10 \times 10 \mathrm{~cm})$, weighing no more than $1.33 \mathrm{Kg}$. The smallest size at CubeSat standard is $1 \mathrm{U}$ while The Aerospace Corporation has launched two 0.5U CubeSat. The certain common variation includes 3U,6U,12U. It supports Moore's Law of advancements in electronics, growing more powerful and sophisticated, lighter, and energy efficient. P-POD design and development were driven by the need for consistency in design and launching of Picosatellite.

\subsection{Detailed Analysis}

The design of the satellite is composed of a cube structure as the name suggests along with an electronic circuitry made with COTS (Commercial of The Shelf) elements. To provide for power requirements it has solar cells on each face, also there are two rechargeable batteries in the centre. Now the batteries split the function into two, one for satellite computer, communications electronics and attitude control system while another for the payload. The

CubeSat has two standard constraints, first being dimensions which should be a ten-centimetre cube and have rub rails for deployment out of the CubeSat launcher. The next requirement is the mass, currently which is one kilogram. This results in a number of limitations and challenges in designing a CubeSat. A 10-centimetre cube of water has a mass of one kilogram. Materials used in structuring the satellite have a significantly higher specific density than water. This limitation requires a creative approach to designing the structure. Currently, 200gm is left for the payload and the satellite has a mass of $800 \mathrm{gm}$ [5]. The total production of CubeSat is to be less than one thousand dollars. After all the design should be compact and simple.

Now considering the electronic components of the system, they should have some radiation data associated with them. For example, Motorola using the MOSAIC process manufactures the transmitter and receiver integrated circuits. This process is known to produce radiation tolerant devices [5]. Next is low power consumption. One-watt power is available. Bussmann power is specified to be about 250 mill watts. Since each face of the satellite has eight solar cells, a parallel combination of two series combination of four cells. Along with this, each face is also connected in parallel. The system is so designed that even if one solar cell fails then also there are still four cells active. This way redundancy is 
introduced which is also present in the batteries.

Multipurpose components are used to reduce the number of components and structural mass. For example, the solar cells. They provide power and also acts as sun sensors. The printed circuit boards with the solar cells also have a coil pattern on the opposite side for use as the magnetorquer coils. The circuit board containing the satellite processor, payload and communications will also be the structural members. IIC bus developed by Philips Semiconductor provides a simple serial interface to the payload and forms the communication system. Four digital bidirectional lines are available to the payload. The payload is also provided with two analog to digital converter inputs along with a power control signal. A payload reset signal is also there The receiver audio signal is provided to the payload. This allows the payload to monitor received signals. The user can directly communicate through the receiver. The payload also has access to the transmitter modulation signal.

\section{SOURCES OF HEAT FOR GENERAL SPACE SYSTEM}

\subsection{Sun (Direct Solar Energy)}

Sun is the most constant source of heat and light energy. Most of the spacecraft depend on solar radiation as the main source for heating and power. Depending upon Earth's distance from the Sun, the intensity of sunlight reaching Earth varies approximately $\pm 3.5 \%$ since Earth has an elliptical orbit.

Electromagnetic radiation from the sun that falls on a unit area of the surface normal to the line from the Sun, per unit time, outside the atmosphere, at one astronomical unit (AU) is called Solar constant or Irradiance.

At $1 \mathrm{AU}$ the apparent diameter of the Sun is $0.5^{\circ}$. This implies that at distance closer to Earth parallel rays of the sun are to be considered while at distance closer to sun solar angle should be taken into account.

\subsubsection{Solar radiation}

$G_{s}=1336.1 \pm \frac{10 \mathrm{~W}}{\mathrm{~m}^{2}}($ at $1 \mathrm{AU})$, solar constant,

\subsubsection{For any distance}

$G_{s}\left(d_{s c}-s\right)=G_{s}(1 A U)\left(\frac{d_{E-s}}{d_{s c}-s}\right)^{2}, d_{E-s}$, the distance from

the earth to the sun. [3]

\subsection{Albedo}

Albedo is the sunlight reflected off a planet or moon. For a planet, albedo is the fraction of incident sunlight that is reflected back to space and this is highly variable. Therefore, for Low Earth Orbits (LEO), its influence is higher. The roughness of the surface of the planet diffuses its albedo. When determining the thermal load, albedo loads are considered when the portion of the planet seen by spacecraft is sunlit. Since in LEO changes occur rapidly, mean values are used for thermal design purpose, also the planet is approximated as a reflecting sphere for simplified analysis.

$\dot{Q}_{\text {sun }}=a G_{S} A F_{S C}-\mathrm{p} \cos \emptyset$

The angle $\varphi$ takes into account the fact that the albedo is at a maximum at the sub-solar point and it becomes zero when the planet seen by the spacecraft is in eclipse.

$F_{S C}-\mathrm{p}$ is the view factor between the surface and the planet for $-\frac{\pi}{2} \leq \theta \leq \frac{\pi}{2}$. Where $a$ is albedo coefficient and $\theta$ is the solar zenith angle.

\subsection{Infrared Radiation}

The Earth IR is also known as planetary radiation emitted by it. It is longwave radiation. It is composed of planets radiation and that emitted by atmospheric gases. It lies in the IR region of the spectrum. Thermal energy can be characterized by means of the planets black-body equivalent temperature.

If the albedo coefficient, a, is known, the planet's equivalent black-body temperature can be obtained by equating the solar energy absorbed by the planet to the emitted energy

$$
G_{s} \pi \mathrm{R}_{\mathrm{p}}^{2}(1-\sigma)=4 \pi \mathrm{R}_{\mathrm{p}}^{2} \mathrm{P}^{4}
$$

Where $\sigma$ is the Stefan-Boltzmann constant and $R p$ is the radius of the planet. Note that the resulting temperature is independent of the value of $\mathrm{Rp}$, as can be seen in the equation.

\section{APPLICATIONS}

\subsection{P-PODS and Development}

P-Pods stands for Poly-Picosatellite Orbital Deployer was started as a collaboration between California Polytechnic State University, San Luis Obispo and the Space Systems Development Laboratory in 1999 at Stanford University.

It is a tubular designed box mainly made up of aluminium with spring assisted ejection. This innovation took place with reference to the high demand and efficient deployment of CubeSats. Also, the need for consistency in the design and launching of picosatellite class CubeSat systems drove the design and development of the P-POD. The mechanism which controls the deployment of the CubeSat is of non-explosive nature which up to a good extent minimizes the shock to the vehicle and satellites [4]. The P-POD has three versions Mk$1, \mathrm{Mk}-2$, and Mk-3.

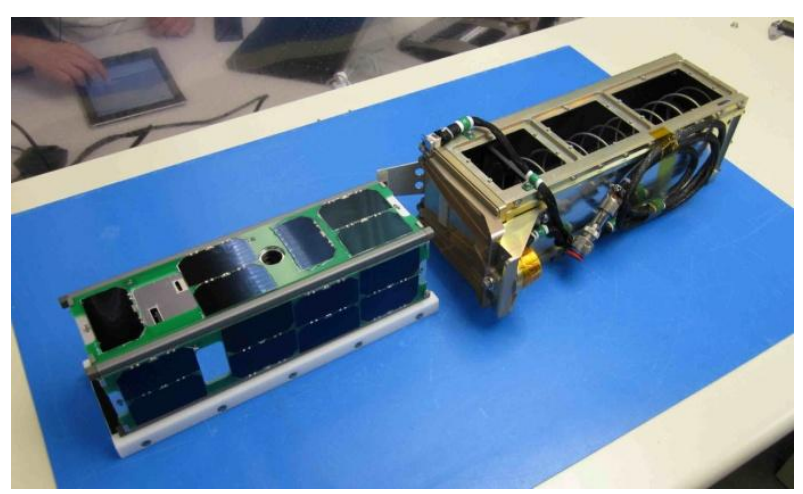

Fig. 1: Poly Picosatellite Orbital Deployer

\subsection{RAINCUBE (Radar in CubeSat)}

RAINCUBE is a type of satellite that will send and receive radar signals with the help of a parabolic antenna acting like the main dish that will reflect the signals onto a device called a sub-reflector. This sub-reflector will channel them into a "feedhorn" and thereafter to the radar circuitry. The RAINCUBE's radar will survey the clouds it's flying over at an altitude of 450 to $500 \mathrm{~km}$ wide radar footprint. Before the RAINCUBE is deployed the antenna needs to fold up into a canister measuring 10 by 10 by 15 centimetres. The radar operates at a frequency of $35.75 \mathrm{GHz}$ which means that it should be precisely deployed so that its shape deviates from perfection by no more than 200 micrometres [1]. 


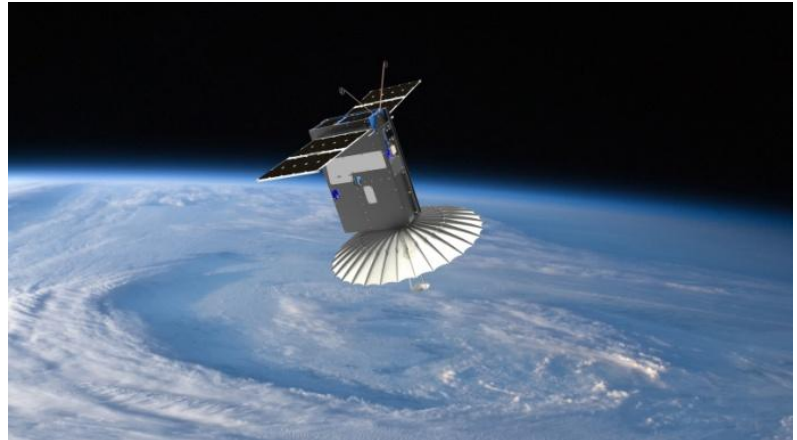

Fig. 2: RAINCUBE satellite

\subsection{MARCO (Mars Cube One)}

Marco contains a flat antenna also known as the reflect array, which consists of a three-part panel that flips out from the side of the spacecraft and opens under the power of spring-loaded hinges. When the panel flips away from the body of the spacecraft, the feedhorn of the antenna also pops and then rotates around an off-the-shelf connector. This was confined to only 4 percent of the spacecraft payload's volume. The antenna's flat surface is dotted with a reflective pattern so that it can copy the actions of a parabolic antenna and concentrate signals in the direction of Earth.

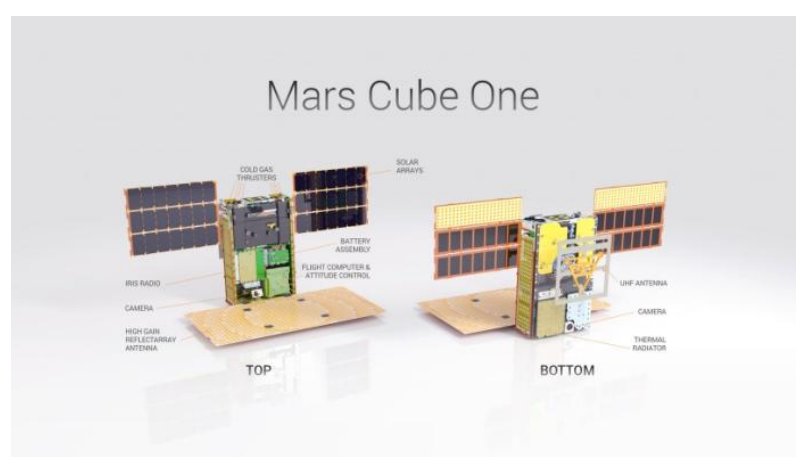

Fig.3: MARCO (Mars Cube One)

\section{DEVELOPED CUBE-SATS}

\subsection{TURKSAT}

It was a preliminary effort by the Turks to design a native communication satellite named TURKSAT [6]. The problem occurred was the payload power requirement which resulted in temperatures exceeding $100 \mathrm{C}$. The remedy to this was then developed to remove heat resulting from a power amplifier. The first model of a TURKSAT was TURKSAT-3USAT which is a three-unit CubeSat.

\subsection{MAKERSAT}

Talking of $1 \mathrm{U}$ multi-project satellite, supporting up to four science payloads, MAKERSAT was developed. The materials used were expected to undergo mass loss due to monoatomic oxygen radicals. This made the MAKERSAT have launch problems.

One way to avoid such problems is to use "stash and deploy" technique proposed by NanoRacks and Made in Space2 [2]. MAKERSAT is the first CubeSat designed majorly for additive manufacturing assembled by ISS crew. It is an open source as well as a multi-user space research platform available to the researchers and students and start-ups. MAKERSAT is assembled in two versions, MAKERSAT-0 and MAKERSAT-1.
MAKERSAT- 0 is manufactured and assembled on the terrestrial surface and launched into sun-synchronous polar orbit.

MAKERSAT-1 is manufactured, assembled, and launched completely from the ISS. The computational power is provided by an ultra-low power MSP430FR6989 microcontroller with $128 \mathrm{kB}$ of FLASH memory.

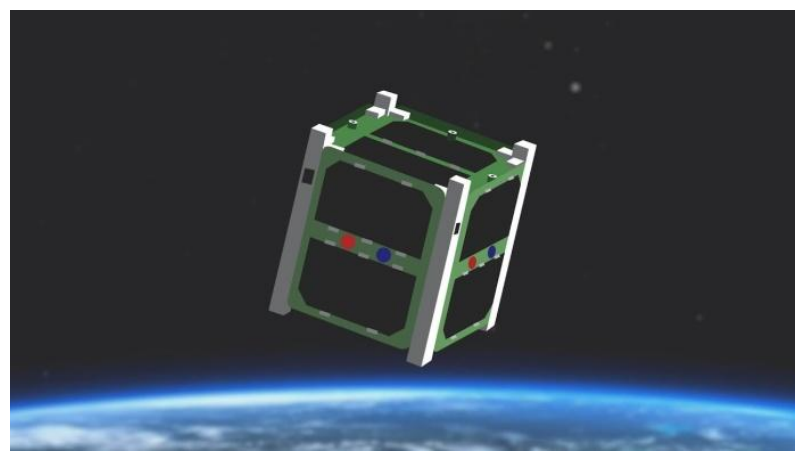

Fig. 4: MAKERSAT-0

\subsection{ECUADORIAN CIVILIAN SPACE AGENCY}

The NEE-01 PEGASUS was the first satellite developed by the Ecuadorian Civilian Space Agency. The demand for very thin, deployable, multi-panel solar arrays, able to fold in no more than $6 \mathrm{~mm}$ height as a spacecraft of $1 \mathrm{U}$ CubeSat design and the P-POD restrictions was fulfilled.

It resulted in a device that used no compound hinges for release and deploy that not only met the mission requirements of the agency but actually surpassed the use of titanium scaffold coupled with memory shape alloys in such a geometry that naturally provide the deployment force needed and the use of sun's heat to activate the memory.

\section{SALIENT FEATURES}

\subsection{Cheap and Convenient}

CubeSats offer the same capabilities as of traditional satellites while costing significantly less. According to 'The Conversation', while the initial cost of traditional satellites can be hundreds of millions of dollars, a CubeSat can be built for around one hundred thousand dollars approximately with the same specifications. Further, nanosatellites are more convenient to build. Due to their low cost and less stringent standards of regulation. They can be built faster.

\subsection{Research and Development}

Easy to prototype and build, CubeSats are known for its research purposes. It's cost effective nature provides a great platform for countries having financial issues. In addition to that this platform provides with the best way to demonstrate satellite projects at an intermediate level of studies.

\subsection{Integrated with Modern and Advanced Technology}

A CubeSat is integrated with the latest sensorics and attachment in order to increase the functionality of the satellite for its functions as well as for its control. These sensors are composed with satellite to measure its position, detect pressure, etc. These technologies provide CubeSats with a wealth of resources to work with.

Being low cost, multiple satellites can be launched into the Low-Earth orbit. The frequency of these satellites passing 
through a specified geographical area is more than the normal satellite's area. The high flexibility of CubeSats and the ability to provide multiple services to different customers enables the opportunity for the enhanced usage of internet facilities and GPS services.

\section{LIMITATIONS}

The heading of a section should be in Times New Roman 12point bold in all-capitals flush left with an additional 6-points CubeSats have the ability of high endurance and better coverage ability but still, the area where it lags the most is its life span. The approximate life span is of 2 to 3 years which is very less when compared to the life span of normal satellites.

Payload capacity is another factor which makes the CubeSat open for the research purpose in this particular field. Being a small-scale satellite, this offers a very less payload capacity and thus cannot be used for heavy missions.

\section{CONCLUSIONS}

CubeSats are a great source of learning which helps in research related activities as well. The exploration level with the use of these devices acts as a major activity. A conventional satellite weighing far more than that can for sure carry many instruments but CubeSat is a cheap and attractive option for a rather specific goal.

\section{ACKNOWLEDGMENTS}

It is a genuine pleasure to express our deep sense of thanks to our mentor Dr. Neelesh Kumar Gupta, Professor (E.C.E. Department) at Ajay Kumar Garg Engineering College, Ghaziabad. His dedication and overwhelming attitude towards us has been solely responsible for completing our work.

His views greatly assisted us during this research along with comments on earlier versions of the manuscript. Any errors are our own and should not tarnish the reputation of the institution.

\section{REFERENCES}

[1] Nacer E. Chahat. (2018). New Antennas Will Take CubeSats to Mars and Beyond: By packing big antennas into tiny satellites, JPL engineers are making space science cheap [Online] Available at https://spectrum.ieee.org/aerospace/satellites/newantennas-will-take-cubesats-to-mars-and-beyond.

[2] Grim, Braden \& Kamstra, Mitch \& Ewing, Aaron \& Nogales, Connor \& Griffin, Joshua \& Parke, Stephen. (2016). MakerSat: A CubeSat Designed for In-Space Assembly.

[3] Adam Bin Mohd Fikri, Muhammad Fais Haikal Bin Mohd Isa and Abeeb Fajobi Olamilekan. Thermal Control System in CubeSat - Critiques on Multi-Layer Insulation and Louver as Alternative.

[4] Ryan Nugent, Riki Munakata, Alexander Chin, Roland Coelho, Dr. Jordi Puig-Suari. (2000). The CubeSat: The Picosatellite Standard for Research and Education.

[5] Mr. Hank Heidt, Prof. Jordi Puig-Suari, Prof. Augustus S. Moore, Prof. Shinichi Nakasuka, Prof. Robert J. Twiggs. (2000) CubeSat: A new Generation of Picosatellite for Education and Industry Low-Cost Space Experimentation.

[6] Prof.Dr. Alim Rustem Aslan, Dr. Bulent Yagci, Ertan Umit, Mustafa Erdem Bas, Mehmet Şevket Uludağ, Okan Emre Özen, Murat Süer, Ahmet Sofyall, Assist. Prof. Dr. Cuma Yarim. (2013). Lessons Learned Developing a 3U Communication CubeSat. 